SCIREA Journal of Clinical Medicine

ISSN: 2706-8870

http://www.scirea.org/journal/CM

October 10, 2021

SCIREA

Volume 6, Issue 6, December 2021

\title{
The feasibility of combined thermal and nonthermal e ndovenous ablation in comparison of nonthermal ablat ion for superficial vein insufficiency from single center
}

KJ Choi ${ }^{1}$, HJ Jun², Myunghee Yoon ${ }^{3, *}$

${ }^{1}$ Department of cardiovascular surgery, Khatlon state medical university, Tajikistan

${ }^{2}$ Department of vascular surgery, Jun's vascular clinic, Busan, Republic of Korea

${ }^{3}$ Department of Surgery, Pusan National University Hospital, Republic of Korea

"Corresponding Author:

Myunghee Yoon

Associate Professor

Department of Surgery

Pusan National University Hospital

Biomedical Research Institute

179 Gudeok-Ro, Seo-Gu, Busan, Busan, 49241 Republic of Korea

TEL : +82-51-240-7238, FAX : +82-51-247-1365

Email : ymh@pusan.ac.kr

\section{Abstract}

Background: Nonthermal endovenous ablation has been needed high amount of scleros ant for the treatment of saphenous vein insufficiency. However, its safe amount has $n$ 
ot been known clearly and nonthermal edovenous ablation should be performed avoidi ng thrombotic complication. This study is to evaluate the feasibility of the combined nonthermal and thermal endovenous ablation technique to reduce the amount of sclero sant in comparison of nonthermal endovenous ablation monotherapy.

Methods: Between June 2018 and May 2020, a total of 327 patients diagnosed with s uperficial vein insufficiency were evaluated retrospectively. 130 patients were includedi n Nonthermal mechanochemical ablation (MOCA, Group I) monotherapy, 197 patient $\mathrm{s}$ in combined thermal and nonthermal endovenous ablation treatment (EVLA with $\mathrm{M}$ OCA, Group II) from one surgeon of single center. Combined EVLA and MOCA ther apy was mostly performed for patients who had varicose veins in 3 or more veins.

Results: The amount of STD used per number of legs was $5.5 \pm 2.05 \mathrm{~mL}, 4.51 \pm 1.2 \mathrm{~m}$ L in Group I, Group II respectively $(p<0.001)$. The amount of STD used per number of veins was $4.77 \pm 1.91 \mathrm{~mL}, 3.12 \pm 1.02 \mathrm{~mL}$ in Group I, Group II respectively $(\mathrm{p}<0.00$ 1). Recanalization rates were $0 \%(0 / 130)$ within 52 weeks, $2.31 \%(3 / 130)$ after 52 we eks in Group I, 5.58\% (11/197) within 52 weeks, 6.60\% (13/197) after 52 weeks inG roup II, it was not statistically significant. Complications within 4 weeks Complication rates were $3.84 \%, 7.11 \%$ in Group I, Group II respectively

Conclusions: Combined EVLA and MOCA procedure was effective for those who had 3 or more varicose veins insufficiency. The varicose vein anatomical occlusion at $1 \mathrm{y}$ ear, patient satisfaction and complication rates were included.

Keywords: Chronic venous insufficiency, Thermal endovenous laser ablation, non-the rmal mechanochemical ablation, sclerosant, occlusion

\section{Introduction}

Chronic superficial venous insufficiency is very common in modern society. People w ho are affected has been shown a decreased quality of life (QoL). In the past, the ai $\mathrm{m}$ of the treatment was on removal of the pathological vein. Since the sclerosant suc $\mathrm{h}$ as sodium tetradecyl sulfate (STD) has been introduced, the endovenous ablation be came the major modality for treatment of venous insufficiency in lower leg. In the pa st decades, endovenous laser ablation (EVLA), radiofrequency ablation (RFA), ultrasou 
nd-guided foam sclerotherapy (USFS) and mechanochemical ablation (MOCA) have be en gained popularity.

Many trials have been reported these treatment methods are different in the quality of life and the result of surgery. A randomized trial treatment for varicose veins revealed that laser ablation was better than foam sclerotherapy in disease-specific quality of lif e 5 years after treatment [1]. A randomized clinical trial showed that all treatments $\mathrm{w}$ ere acceptable in efficiency and a higher failure rate was shown after foam sclerother apy, but postoperative pain and low quality of life were in endovenous laser and strip ping comparing to radiofrequency ablation and foam sclerotherapy [2]. Endovenous th ermal ablations such as laser ablation and radiofrequency ablation have limits related $\mathrm{t}$ hermal injury to adjacent tissue which affects the quality of life postoperatively. Nont hermal mechanochemical endovenous ablation with clarivien ${ }^{\circledR}$ avoids thermal injury $b$ ut considerable amount of sclerosant should be infiltrated to close the proximal portio $\mathrm{n}$ of the greater saphenous vein (GSV). Even though the adequate amount of sclerosa nt is debated, but considering the side effects of sclerosant, it seems better to use a $r$ educed amount of sclerosant.

The aim of this study was to evaluate that concomitant thermal and nonthermal endo venous ablation is safe and feasible for patients who had 3 or more veins of superfic ial vein insufficiency and also and reduces the amount of sclerosant comparing nonthe rmal MOCA monotherapy.

\section{Methods}

Between June 2018 and May 2020, a total of 327 patients diagnosed with superficial vein insufficiency were evaluated retrospectively. Nonthermal mechanochemical ablation (MOCA, Group I) monotherapy was performed on 130 patients, combined thermal an d nonthermal endovenous ablation treatment (EVLA with MOCA, Group II) were perf ormed on 197 patients from one surgeon of single center.

Inclusion criteria were age 18-75 years; symptomatic varicose veins, Clinical Etiologic Anatomic Pathophysiologic (CEAP) class C2-6, GSV incompetence, defined by a refl ux time of more than $0.5 \mathrm{~s}$ on duplex imaging (Linear i18LX probe; TUS-AI700, To shiba Medical Systems, Japan) [3]. The patients were examined in the standing positi 
on, and reflux was measured after manual compression and release of the calf. Bilater al treatment was permitted, provided that both legs had the same treatment during the same operation. Patients with recurrent varicose veins were also included if the GSV was preserved to the groin on duplex imaging.

A light sedative and analgesic (midazolam) were administered intravenously before the procedure in most patients.

The EVLA procedure was performed under duplex guidance with a $1940 \mathrm{~nm}$ GaAIAs laser diode, continuous wave, 600 micrometer fiber (Atoven-1, Diotec, Korea) for all patients. A bare-tip fiber was used for all EVLA treatments. The laser fibere was adv anced until $2 \mathrm{~cm}$ below the saphenofemoral junction (SFJ), after which the GSV was ablated during withdrawal of the fiber at $10 \mathrm{~mm}$ per 7-8 sec with 4-7 watt of laser energy. All treatments were performed in an operation room under tumescent local an esthesia, using a normal saline solution of $0 \cdot 1$ percent lidocaine $(\mathrm{N} / \mathrm{S} 500 \mathrm{~mL}+2 \% \mathrm{~L}$ idocaine $25 \mathrm{~mL}$ ). The solution was administered using an infusion pump (Endo-jet, Me sa Medical, Korea) under ultrasound guidance. The aim was to administer $10 \mathrm{ml}$ per cm GSV tumescent anesthesia in the combined Group II.

Clarivein ${ }^{\circledR}$ (Bridgemedica LLC, USA) procedure was performed with two steps of act ion. Mechanical agitation of the vessel endothelium by a rotating catheter tip. A scler osant drug sprayed from the tip of the catheter as it is withdrawn to ensure maximal effect. No tumescent anaesthesia was required. All the patients were positioned supine with the leg slightly flexed and abducted to enhance access to both the GSV and the SSV. A Seldinger technique was used to introduce a $5 \mathrm{Fr}$ introducer sheath into eith er the GSV or SSV under ultrasound guidance and flushed with saline. The ClariVein (R OC infusion catheter (Bridgemedica LLC, USA) tip was inserted through the sheat $\mathrm{h}$ and the tip of the dispersion wire positioned $10 \mathrm{~mm}$ distal to the saphenofemoral $\mathrm{j}$ unction or saphenopopliteal junction. The sheath was withdrawn to just beyond the pu ncture site to prevent activation of the probe within the sheath. Wire rotation was act ivated for a few seconds to induce spasm of the proximal vein. With the wire contin uing to rotate, infusion of the sclerosant was started simultaneously with catheter pull back. The activated catheter was steadily withdrawn at $1 \mathrm{~cm}$ every 7 to $10 \mathrm{~s}$. The sc lerosant used was $2.0 \%$ liquid sodium tetradecyl sulphate (STD) [4].

Generally, $0.1 \mathrm{ml}-0.2 \mathrm{ml}$ of sclerosant is injected every $1 \mathrm{~cm}$ pullback on the cathete 
r. A completion duplex ultrasound was performed after the procedure to confirm the $\mathrm{p}$ atency of the common femoral vein and the deep venous system.

After the procedure, the leg was wrapped in sterile absorbent bandages and covered with a cohesive compression bandage for $48 \mathrm{~h}$. Patients were then instructed to use a compression stocking to the groin for 2 weeks. No specific analgesia was prescribed. All patients were encouraged to resume work and normal activity as soon as they we re able

The study design was approved by the Institutional Review Board of Pusan National University Hospital (PNUH No. 2109-011-107) and was conducted in accordance witht he Declaration of Helsinki.

\section{Follow-up}

Patients were asked to document the level of peri and postprocedural pain. A medianf ollow-up period was 1 year. Patients visited on clinic at two weeks, two months, six months, and 1 year and an ultrasound study and clinical exam were performed. Color duplex scan was performed scanning the full length of the treated vein testing for $\mathrm{c}$ ompressibility and reflux. A successfully obliterated vein was solid with no visible lu men and could not be compressed, and there was no flow on color duplex and Valsal va.

\section{Statistical analysis}

Continuous variables are reported as mean and standard deviation and categorical vari ables as absolute number and percent, unless stated otherwise. Continuous data were c ompared using the Student $\mathrm{t}$ test for parametric and non-parametric data, respectively. Categorical data were compared using the Chi-square or Fisher exact tests. Statistical significance was assumed at $\mathrm{p}<0.05$. The statistical analyses were performed using R 3.6.0.

\section{Results}

Between June 2018 and May 2020, a total of 327 patients diagnosed with superficial 
vein insufficiency were evaluated retrospectively. Nonthermal mechanochemical ablatio n (MOCA, Group I) monotherapy was performed on 130 patients, combined thermal and nonthermal endovenous ablation treatment (EVLA with MOCA, Group II) were $p$ erformed on 197 patients from one surgeon of single center.

Clinical characteristics were described in Table 1. Mean age was 51.89 $\pm 12.95,54.96 \pm$ 12.53, female was $74.6 \%, 62.9 \%$ in Group I, Group II respectively. According to CE AP Classification, C2-C4 were 99.23\%(129/130), 98.47\%(194/197) in Group I, Group II respectively.

Table1. Clinical characteristics between MOCA (Group I) and combined EVLA and MOCA (Group II)

\begin{tabular}{|c|c|c|c|c|}
\hline \multirow[b]{2}{*}{ Variables } & Overall & Group I & Group II & \multirow[b]{2}{*}{ P-value } \\
\hline & $(\mathrm{n}=327)$ & $(n=130)$ & $(n=197)$ & \\
\hline Age & $53.74 \pm 12.77$ & $51.89 \pm 12.95$ & $54.96 \pm 12.53$ & 0.034 \\
\hline Female/Male (\%) & $221(67.6 / 106(32.4)$ & $97(74.6) / 33(25.4)$ & $124(62.9) / 73(37.1)$ & 0.037 \\
\hline \multicolumn{5}{|l|}{ CEAP Classification } \\
\hline $\mathrm{C} 2$ & $163(49.85)$ & $63(48.46)$ & $100(50.76)$ & \\
\hline $\mathrm{C} 3$ & $140(42.81)$ & $61(46.92)$ & $79(40.10)$ & \\
\hline $\mathrm{C} 4$ & $20(6.12)$ & $5(3.85)$ & $15(7.61)$ & 0.385 \\
\hline $\mathrm{C} 5$ & $2(0.61)$ & $0(0)$ & $2(1.02)$ & \\
\hline C6 & $2(0.61)$ & $1(0.77)$ & $1(0.51)$ & \\
\hline Diameter GSV & $8.27 \pm 2.62$ & $7.72 \pm 2.27$ & $8.63 \pm 2.77$ & $<0.001$ \\
\hline Complication (\%) & $19(5.81)$ & $5(3.84)$ & $14(7.11)$ & 0.321 \\
\hline \multicolumn{5}{|l|}{ Recanalization (\%) } \\
\hline Early (Within 52 weeks) & $11(3.36)$ & $0(0)$ & $11(5.58)$ & \\
\hline & & & & 0.247 \\
\hline Delayed (After 52 weeks) & $16(4.89)$ & $3(2.31)$ & $13(6.60)$ & \\
\hline Total $(\%)$ & $27(8.25)$ & $3(2.31)$ & $24(12.18)$ & 0.003 \\
\hline
\end{tabular}




\begin{tabular}{|c|c|c|c|c|}
\hline \multicolumn{5}{|c|}{ Number of legs $(\%)$} \\
\hline 1 & $88(26.91)$ & $70(53.85)$ & $18(9.14)$ & \multirow[b]{2}{*}{$<0.001$} \\
\hline 2 & $239(73.09)$ & $60(46.15)$ & 179(90.86) & \\
\hline \multicolumn{5}{|c|}{ Number of veins (\%) } \\
\hline 1 & $68(20.79)$ & $55(42.3)$ & $13(6.6)$ & \\
\hline 2 & $103(31.50)$ & $55(42.3)$ & $48(24.37)$ & \\
\hline 3 & $98(29.97)$ & $14(10.78)$ & $84(42.64)$ & $<0.001$ \\
\hline 4 & $58(17.74)$ & $6(4.62)$ & $52(26.39)$ & \\
\hline Total STD (mL) & $8.14 \pm 2.64$ & $7.56 \pm 2.71$ & $8.52 \pm 2.54$ & $<0.001$ \\
\hline STD/Leg (mL) & $4.91 \pm 1.69$ & $5.5 \pm 2.05$ & $4.51 \pm 1.27$ & $<0.001$ \\
\hline STD/Vein (mL) & $3.77 \pm 1.65$ & $4.77 \pm 1.91$ & $3.12 \pm 1.02$ & \\
\hline
\end{tabular}

CEAP Clinical Etiologic Anatomic Pathophysiologic (CEAP), STD Sodium tetradecyl sulphate MOCA mechenochemical ablation, EVLA endovenous laser ablation

Diameter GSV was $72 \pm 2.27 \mathrm{~cm}, 8.63 \pm 2.77 \mathrm{~cm}$ in Group I, Group II respectively, it was statistically significant $(\mathrm{p}<0.001)$. Patients with varicose veins in both legs were 46.1 5\%(60/130), 90.86\%(179/197) in Group I, Group II respectively $(\mathrm{p}<0.001)$.

Patients with 3 or more varicose veins were $15.40 \%(20 / 130), 69.03 \%(136 / 197)$ in Gro up I, Group II respectively $(\mathrm{p}<0.001)$.

Totally used sclerosant such as sodium tetradecyl sulfate (STD) was 7.56 $\pm 2.71 \mathrm{~mL}$, $8.52 \pm 2.54 \mathrm{~mL}$ in Group I, Group II respectively $(\mathrm{p}<0.001)$. The amount of STD used per number of legs was $5.5 \pm 2.05 \mathrm{~mL}, 4.51 \pm 1.2 \mathrm{~mL}$ in Group I, Group II respectively $(\mathrm{p}<0.001)$. The amount of STD used per number of veins was $4.77 \pm 1.91 \mathrm{~mL}, 3.12 \pm 1$. $02 \mathrm{~mL}$ in Group I, Group II respectively $(\mathrm{p}<0.001)$.

Complication rates were $3.84 \%, 7.11 \%$ in Group I, Group II respectively (Table 2). R ecanalization was $0 \%(0 / 130)$ within 52 weeks, 2.31\% (3/130) after 52 weeks in Gro up I, 5.58\% (11/197) within 52 weeks, 6.60\% (13/197) after 52 weeks in Group II, it was not statistically significant (Table 3 ). 
Table 2. Type of complications between MOCA (Group I) and combined EVLA and MOCA (Group II)

\begin{tabular}{lll}
\hline \multirow{2}{*}{ Type of Complication } & Group I $(\mathrm{n}=130)$ & Group II $(\mathrm{n}=197)$ \\
\cline { 2 - 3 } & within 4 weeks & within 4 weeks \\
\hline edema & 0 & 2 \\
hemoglobinuria & 1 & 1 \\
local skin infection & 0 & 1 \\
pain along vein & 0 & 1 \\
pigmentation & 2 & 0 \\
pigmentation thrombophebitis & 0 & 8 \\
rash, thrombophlebitis & 2 & 1 \\
wound oozing & 0 & $14(7.11)$ \\
\hline Total (\%) & $5(3.85)$ & \\
\hline
\end{tabular}

MOCA mechenochemical ablation, EVLA endovenous laser ablation;

Table 3. Type of recanalization between MOCA (Group I) and combined EVLA and MOCA (Group II)

\begin{tabular}{|c|c|c|c|c|}
\hline \multirow{2}{*}{ Type of of recanalization } & \multicolumn{2}{|c|}{ Group I $(n=130)$} & \multicolumn{2}{|c|}{ Group II (n=197) } \\
\hline & Early & Delayed & Early & Delayed \\
\hline both gsv prox & 0 & 0 & 3 & 1 \\
\hline both gsv thigh & 0 & 0 & 1 & 0 \\
\hline both ssv prox & 0 & 1 & 0 & 0 \\
\hline lt gsv bk & 0 & 0 & 0 & 1 \\
\hline It gsv thigh & 0 & 0 & 0 & 3 \\
\hline It gsv thigh, lt ssv & 0 & 0 & 0 & 1 \\
\hline lt $s f j$ & 0 & 1 & 0 & 0 \\
\hline lt ssv & 0 & 0 & 2 & 2 \\
\hline lt ssv, gsv bk & 0 & 0 & 1 & 0 \\
\hline rt gsv bk, lt gsv thigh & 0 & 0 & 0 & 1 \\
\hline rt gsv thigh & 0 & 0 & 3 & 1 \\
\hline rt gsv, lt ssv & 0 & 1 & 0 & 0 \\
\hline $\mathrm{rt} \mathrm{ssv}$ & 0 & 0 & 1 & 3 \\
\hline
\end{tabular}


MOCA mechenochemical ablation, EVLA endovenous laser ablation

gsv great saphenous vein, ssv small saphenous vein, sfj saphenofemoral junction, bk below knee

\section{Discussion}

In this study, combined EVLA and MOCA therapy was mostly performed for patients who developed varicose veins in 3 or more veins. After 1year follow-up after surgery, the insufficient varicose vein occlusion rate is high, and the amount of STD used wa $\mathrm{s}$ also reduced per number of legs and per number of veins. Bilateral procedures can be successfully performed, and these are well tolerated as can multiple veins in the $b$ oth leg. MOCA monotherapy was also excellent in the patient's quality of life and va ricose vein occlusion rate with the usual amount of STD used. ClariVein can be used to ablate long and short saphenous varicose veins on a walk-in-walk-out basis.

The volume of sclerosant used was not predetermined but adjusted on a case-by-case basis by continuous duplex monitoring of the mechanical and chemical effect to ensue spasm and collapse of the vein, while not exceeding the safe dose of sclerosant. Gen erally, $0.1 \mathrm{ml}-0.2 \mathrm{ml}$ of sclerosant is injected every $1 \mathrm{~cm}$ pullback on the catheter. $\mathrm{V}$ ein diameter was determined by duplex ultrasound measurement from the widest part of the treated vein in the supine position excluding the first $2 \mathrm{~cm}$ of vein and any lo calized venous blowouts.

When adhering to safe dosage levels, sclerosants with higher concentrations potentially limit the extent of treatment. By Lam, at 6 weeks post-treatment duplex ultrasound sh owed that $100 \%, 96.4 \%$ and $56.5 \%$ were occluded using $2 \%$ Polidocanol liquid, 3\% Polidocanol liquid and 1\% Polidocanol microfoam in the mechano-chemical ablation $r$ espectively [5].

The frequency of recanalization after 2 years from the VenaBlock procedure was signi ficantly higher than after laser treatment (37.2 vs. 8.7\%) [6].

At eight weeks' follow-up, there was only partial obliteration in 13/393 (3.3\%) veins after mechanochemical endovenous ablation with ClariVein ${ }^{\circledR}$ [7].

Recently, a randomized controlled crial of endovenous laser ablation versus mechanoch emical ablation with ClariVein was reported. Both EVLA and MOCA were highly effi cacious in treating superficial vein insufficiency. Both resulted in low procedural pain 
with a short recovery time. Axial occlusion rates were higher after EVLA [8-10]. The outcomes were intra-procedural ablation pain scores and anatomical occlusion at 1 yea r. Post-procedural pain, venous clinical severity score, quality of life, patient satisfacti on and complication rates were included.

In our study, both combined EVLA and MOCA (Group II), MOCA monotherapy (Gro up I) were highly efficacious in treating superficial venous insufficiency. Patients impr oved significantly in terms of disease severity, symptoms, low procedural pain with as hort recovery time. About recanalization, of 24 patients (Group II) who had recanaliza tion, 16 patients who used 4 watt of laser energy, 7 patients who used 5 watt of las er energy, and 1 patient who used 6 watt of laser energy during EVLA. Recanalizatio $\mathrm{n}$ rate was low in the case of combined procedure using 7 watt of laser energy. Lase r energy with 7watt has been mainly used since May 2018.

Tumescent anesthesia is currently required for endothermal ablation technique and carr ies the risk of thermal-related complications such as neuralgia, skin burn and prolonge $\mathrm{d}$ pain $[11,12]$. The insertion of tumescence itself can also be painful and cause comp lications. The ClariVein occlusion catheter is a relatively minimally invasive approach with a liquid sclerosant infusion. It has the advantage of eliminating the need for tum escent anesthesia and the risks of heat related injury to the surrounding tissue and str uctures. It has been shown to be safe and efficacious in its initial trials $[13,14]$ not o nly for the great saphenous vein but also for the short saphenous vein $[15,16]$. Proce dure times and intra/post-procedural pain scores have better than for RFA and EVLA $[17,18]$. Kwon et al recommended that caution should be exercised when the epifascia 1 GSV tributary is treated during the ClariVein procedure because of its hyperpigment ation as a complication [19].

\section{Conclusion}

The occlusion rate of GSV with EVLA and MOCA combination therapy was effective, and the total amount of STD per number of lesion veins and legs could be reduced, thereby reducing side effects.

Author Contributions: Conceptualization, KJ Choi, HJ Jun, Myunghee Yoon; method 
ology, HJ Jun; Data collection and processing, KJ Choi, Myunghee Yoon; formal ana lysis, Myunghee Yoon; literature search, KJ Choi, HJ Jun, Myunghee Yoon; writing —original draft preparation, KJ Choi, Myunghee Yoon writing-review and editing, Myunghee Yoon.

All authors have read and agreed to the published version of the manuscript.

Institutional Review Board Statement: The study was conducted according to the gu idelines of the Declaration of Helsinki. The study design was approved by the Institut ional Review Board of Pusan National University Hospital (PNUH No. 2109-011-107).

\section{References}

[1] Julie Brittenden, David Cooper, Maria Dimitrova, Graham Scotland, Seonaidh C. Cotton, et al. Five-Year Outcomes of a Randomized Trial of Treatments for Varicose Veins. N Engl J Med 2019; 381, 912-22

[2] L. H. Rasmussen, M. Lawaetz, L. Bjoern, B. Vennits, A. Blemings et al. Randomized clinical trial comparing endovenous laser ablation, radiofrequency ablation, foam sclerotherapy and surgical stripping for great saphenous varicose veins. British J of Surg 2011, 98, 1079-1087

[3] Eklof B, Rutherford RB, Bergan JJ, Carpentier PH, Gloviczki P, Kistner RL et al. Revision of the CEAP classification for chronic venous disorders: consensus statement. $\mathrm{J}$ Vasc Surg 2004, 40, 1248-1252.

[4] Elias S and Raines JK. Mechanochemical tumescentless endovenous ablation: final results of the initial clinical trial. Phlebology 2012; 27: 67-72.

[5] Y L Lam, Irwin M Toonder, Cees H A Wittens. Clarivein ${ }^{\circledR}$ mechano-chemical ablation an interim analysis of a randomized controlled trial dose-finding study Randomized Controlled Trial Phlebology 2016, 31(3), 170-176

[6] Justyna Wilczko, Cezary Szary, Dominika Plucinska and Tomasz Grzela. Two-Year Follow-Up after Endovenous Closure with Short-Chain Cyanoacrylate versus Laser Ablation in Venous Insufficiency. J Clin Med. 2021, 10, 628

[7] TY Tang, JW Kam and ME Gaunt. ClariVein ${ }^{\circledR}$ - Early results from a large single-centre series of mechanochemical endovenous ablation for varicose veins. Phlebology 2017, 32(1), 6-12

[8] Abduraheem Hussein Mohamed, Y Clement Leung, Tom Wallace, George Smith, Daniel Carradice, and Ian Chetter. A Randomized Controlled Trial of Endovenous Laser Ablation Versus Mechanochemical Ablation With ClariVein in the Management of Superficial Venous Incompetence (LAMA Trial). Annals of Surgery 2021, 273, e188e195

[9] Clement C. M. Leung, Daniel Carradice, Tom Wallace and Ian C. Chetter. Endovenous laser ablation versus mechanochemical ablation with ClariVein ${ }^{\circledR}$ in the management of superficial venous insufficiency (LAMA trial): study protocol for a randomised 
controlled trial. Trials 2016, 17, 421

[10] Julie Brittenden, David Cooper, Maria Dimitrova, Graham Scotland, Seonaidh C. Cotton. Five-Year Outcomes of a Randomized Trial of Treatments for Varicose Veins. N Engl J Med 2019, 381, 912-922

[11] Sichlau MJ and Ryu RK. Cutaneous thermal injury after endovenous laser ablation of the great saphenous vein. J Vasc Interv Radiol 2004, 15, 865-867.

[12] Van Den Bos RR, Neumann M, De Roos KP, et al. Endovenous laser ablation-induced complications: review of the literature and new cases. Dermatol Surg 2009, 35, 1206 1214.

[13] Elias S and Raines JK. Mechanochemical tumescentless endovenous ablation: final results of the initial clinical trial. Phlebology 2012, 27, 67-72.

[14] van Eekeren RR, Boersma D, Elias S, et al. Endovenous mechanochemical ablation of great saphenous vein incompetence using the ClariVein device: a safety study. J Endovasc Ther 2011, 18, 328-334.

[15] Boersma D, van Eekeren RR, Werson DA, et al. Mechanochemical endovenous ablation of small saphenous vein insufficiency using the ClariVein device: one-year results of a prospective series. Eur J Vasc Endovasc Surg 2013, 45, 299-303.

[16] Deijen CL, Schreve MA, Bosma J, et al. Clarivein mechanochemical ablation of the great and small saphenous vein: early treatment outcomes of two hospitals. Phlebology 2016, 31, 192-197.

[17] Vun S, Rashid S, Blest N and Spark J. Lower pain and faster treatment with mechanicochemical endovenous ablation using ClariVein. Phlebology 2014, 30, 688-692.

[18] Bootun R, Lane T, Dharmarajah B, et al. Intra-procedural pain score in a randomised controlled trial comparing mechanochemical ablation to radiofrequency ablation: the multicentre venefit versus ClariVein for varicose veins trial. Phlebology 2014, 31, 61-65

[19] Yong Wonn Kwon, Sang Woo Park, Jin Ho Hwang, Hyemin Jang, Jeeyoung Min, et al. Feasibility and short-term complication rate of mechanochemical ablation for epifascial tributaries of incompetent great saphenous veins. J Vasc Surg Venous Lymphat Disord 2021, 9(4), 925-931 